\title{
Characterization of Sporadic Creutzfeldt-Jakob Disease and History of Neurosurgery to Identify Potential Iatrogenic Cases
}

\author{
Tsuyoshi Hamaguchi, Kenji Sakai, Atsushi Kobayashi, Tetsuyuki Kitamoto, Ryusuke Ae, \\ Yosikazu Nakamura, Nobuo Sanjo, Kimihito Arai, Mizuho Koide, Fumiaki Katada, Masafumi Harada, \\ Hiroyuki Murai, Shigeo Murayama, Tadashi Tsukamoto, Hidehiro Mizusawa, Masahito Yamada
}

We previously reported a phenotype of CreutzfeldtJakob disease (CJD), CJD-MMiK, that could help identify iatrogenic CJD. To find cases mimicking CJDMMiK, we investigated clinical features and pathology of 1,155 patients with diagnosed sporadic CJD or unclassified CJD with and without history of neurosurgery. Patients with history of neurosurgery more frequently had an absence of periodic sharp-wave complexes on electroencephalogram than patients without a history of neurosurgery. Among 27 patients with history of neurosurgery, 5 had no periodic sharpwave complexes on electroencephalogram. We confirmed 1 case of CJD-MMiK and suspected another. Both had methionine homozygosity at codon 129 of the prion protein gene and hyperintensity lesions in the thalamus on magnetic resonance images of the brain, which might be a clinical marker of CJD-MMiK. A subgroup with a history of neurosurgery and clinical features mimicking dura mater graft-associated CJD might have been infected during neurosurgery and had symptoms develop after many years.

Author affiliations: Kanazawa University Graduate School of Medical Sciences, Kanazawa, Japan (T. Hamaguchi, K. Sakai, M. Yamada); Hokkaido University, Sapporo, Japan (A. Kobayashi); Tohoku University Graduate School of Medicine, Sendai, Japan (T. Kitamoto); Jichi Medical University, Shimotsuke, Japan (R. Ae, Y. Nakamura); Tokyo Medical and Dental University, Tokyo, Japan (N. Sanjo); National Hospital Organization Chiba-East Hospital, Chiba, Japan (K. Arai, M. Koide); Kameda Medical Center, Chiba (F. Katada); The University of Tokushima, Tokushima, Japan (M. Harada); International University of Health and Welfare, Narita, Japan (H. Murai); Tokyo Metropolitan Institute of Gerontology, Tokyo (S. Murayama); National Center of Neurology and Psychiatry, Kodaira, Japan (T. Tsukamoto, H. Mizusawa)

DOI: https://doi.org/10.3201/eid2606.181969
Drion diseases are fatal, transmissible neurodegen1 erative disorders characterized by deposition of abnormal prion protein $\left(\mathrm{PrP}^{\mathrm{Sc}}\right)$ in the brain (1). Human prion diseases, which include Creutzfeldt-Jakob disease (CJD), are classified into sporadic, genetic, and acquired forms. Acquired forms can be transmitted from humans to humans, as in cases of iatrogenic CJD or kuru, or from animals or humans to humans, as in cases of variant CJD (vCJD). Thus far, $>400$ cases of iatrogenic CJD have been reported (2). Two major medical procedures, intramuscular injection of cadaveric human growth hormone and cadaveric human dura mater grafting, have caused iatrogenic transmission of $\mathrm{PrP}^{\mathrm{Sc}}$, and 154 patients, $>60 \%$ of cases worldwide of dura mater graft-associated CJD (dCJD), have been identified in Japan $(2,3)$. We previously showed that dCJD could be classified into 2 types, nonplaque and plaque, according to the pathology findings $(4,5)$. Nonplaque-type dCJD shows clinical features identical to those of typical sporadic CJD (sCJD), but plaque-type dCJD is characterized by atypical pathology and clinical features, including slow disease progression, lack of or late occurrence of periodic sharp-wave complexes (PSWCs) on patients' electroencephalograms (EEGs), and plaque formation in the brain $(4,5)$.

Molecular features of plaque type dCJD include methionine (M) homozygosity at codon 129 (129MM) of the prion protein gene (PRNP) and a distinctive type of $\mathrm{PrP}^{\mathrm{Sc}}$ with intermediate electrophoretic mobility $(-20 \mathrm{kDa})$ between type 1 and type 2 , which has been designated as intermediate type (type i $\operatorname{PrP}^{\mathrm{Sc}}$ ) $(6,7)$. Clinicopathology studies of patients with dCJD and experimental transmission studies strongly suggest that the combination of $129 \mathrm{MM}$, type i PrPsc, and kuru plaques (CJD-MMiK) is a distinctive hallmark of 
acquired CJD caused by an infection with the V2 sCJD strain to persons with 129MM (6,8-10). We proposed neuropathology and biochemical criteria to identify CJD-MMiK $(8,9)$. Surprisingly, we discovered 2 cases of diagnosed SCJD that showed clinical, pathologic, and molecular features identical to CJD-MMiK, which might have been iatrogenically transmitted because 1 of the patients had a history of neurosurgery and the other patient was a neurosurgeon (8). These results suggested that CJD-MMiK could be a clue to identify acquired CJD cases among patients with SCJD diagnoses. To identify iatrogenic cases, we investigated clinical features and pathology of patients with diagnosed SCJD and a history of neurosurgery to find features similar to CJD-MMiK.

\section{Methods}

\section{The Patients}

We analyzed patients with suspected prion diseases who were registered by the Creutzfeldt-Jakob Disease Surveillance Committee in Japan during April 1999-February 2016. The surveillance system started in April 1999 and prospectively investigated each patient with a surveillance protocol as previously reported $(5,11,12)$. After obtaining written consent from patients or their families, members of the surveillance committee directly examined the patient and collected data from clinical and pathology records. The surveillance committee reviewed each patient's case files and assessed EEGs, diffusionweighted magnetic resonance imaging (DW-MRI), ELISA of cerebrospinal fluid (CSF), and history of neurosurgery and then discussed each case to determine which patients had prion disease. The study protocol was approved by the medical ethics committees of Kanazawa University, Tokyo Medical and Dental University, and National Center of Neurology and Psychiatry in Japan.

For patients with a history of neurosurgery, we collected information about the underlying disease, including the date and hospital in which neurosurgery was performed. We determined which patients had undergone a dura mater graft by reviewing surgical records, querying the neurosurgeon, or examining autopsy findings.

Members of the committee assessed EEGs and determined whether PSWCs were typical of prion disease or suggested prion disease. Typical cases were deemed positive for prion disease, and the full committee reviewed and discussed suggested cases to decide whether PSWCs were positive or negative for prion disease.
We examined the cerebral cortices, basal ganglia, and thalamus for hyperintensity lesions on DW-MRI. Committee members defined hyperintensities or submitted scans to the committee's neuroradiology expert for assessment. For ELISA, we examined the value of 14-3-3 protein in CSF, as previously reported $(13,14)$. We analyzed $P R N P$ for $\mathrm{M}$ or valine $(\mathrm{V})$ polymorphism in at codon 129 by using the open reading frame after extracting DNA from patient blood, as described earlier (15).

We included definite and probable cases of prion disease in this study and classified them into 4 categories: sporadic, acquired, genetic, and unclassified. We used World Health Organization criteria to diagnose sCJD and iatrogenic CJD (16). We diagnosed dCJD when we confirmed dura mater grafting, and considered cases as unclassified CJD when insufficient information on dura mater grafting were available. When we confirmed that dura mater graft was not performed during neurosurgery, we diagnosed sCJD even if the patient had history of neurosurgery not related to dura mater. We classified the patients with pathologically confirmed diagnoses of SCJD and unclassified CJD into 9 categories according to PRNP polymorphisms, the type of PrPsc, and pathology seen at autopsy. The 9 subtypes were MM1, MM2-cortical, MM2-thalamic, MM2+1 (pathological features of MM2 and MM1 types of sCJD), MV1, MV2, VV1, VV2, and MMiK. We included patients with definite SCJD and those with probable sCJD and genetic results for PRNP, which can distinguish genetic from sporadic prion diseases.

\section{Clinical and Pathology Findings}

We investigated 1,161 cases of SCJD in which no dura mater grafting or other iatrogenic causes were proven. We also investigated 3 patients with unclassified CJD and compared them to patients with and without history of neurosurgery. Among the 1,164 patients, 36 had a history of neurosurgery, but we excluded 9 because they underwent neurosurgeries $\leq 1$ year before or after the onset of CJD. Our study included 27 patients with history of neurosurgery and 1,128 without history of neurosurgery.

\section{Statistical Analyses}

We used the Student $t$ test to compare patients with and without history of neurosurgery, age at onset of CJD, and duration between the onset of CJD and the appearance of akinetic mutism or death. We used the Fisher exact test to analyze sex distribution, negative rate of PSWCs on EEG, and positive rates of 14-3-3 protein and total tau protein (cutoff 1,200 pg/mL) in CSF. We used the $\chi^{2}$ test to analyze distribution of $\mathrm{M}$ and $\mathrm{V}$ polymorphisms. We considered $\mathrm{p}<0.05$ statistically 
Table 1. Comparison of the clinical features of patients with Creutzfeldt-Jakob disease with and without history of neurosurgery*

\begin{tabular}{|c|c|c|c|}
\hline Patient characteristics & Neurosurgery & No neurosurgery & $p$ value \\
\hline Total no. patients & 27 & 1,128 & \\
\hline \multicolumn{4}{|l|}{ Sex, no. (\%) } \\
\hline $\mathrm{F}$ & $17(63.0)$ & $647(57.4)$ & 0.353 \\
\hline M & $10(37)$ & $481(42.6)$ & \\
\hline Age at onset, $\mathrm{y} \pm \mathrm{SD}$ (range) & $71.0 \pm 8.8(49-88)$ & $68.7 \pm 9.6(30-91)$ & 0.217 \\
\hline Disease duration, $\mathrm{mo} \pm \mathrm{SD}$ (range) $\dagger$ & $6.1 \pm 7.8(1-28)$ & $6.7 \pm 12.0(0-171)$ & 0.823 \\
\hline Duration from neurosurgery to onset of CJD, $y \pm S D$ (range) & $15.0 \pm 9.1(1-35)$ & & \\
\hline \multicolumn{4}{|l|}{ Polymorphism at codon 129 of prion protein gene, no. (\%) } \\
\hline MM & $25(92.6)$ & $1,101(97.6)$ & 0.138 \\
\hline MV & $2(7.4)$ & $22(1.9)$ & \\
\hline VV & 0 & $5(0.4)$ & \\
\hline Negative rate of PSWCs on EEG, no. (\%) & $5(18.5)$ & $64 / 1,121(5.7)$ & 0.019 \\
\hline 14-3-3 protein in CSF, no. tested/no. positive (\%) & $20 / 22(90.9)$ & $675 / 803(84.1)$ & 0.300 \\
\hline Tau protein in CSF, cutoff $1,200 \mathrm{pg} / \mathrm{mL}$, no. tested/no. positive (\%) & $13 / 14(92.9)$ & $503 / 567(88.7)$ & 0.523 \\
\hline
\end{tabular}

significant. We performed statistical analyses by using SPSS Statistics 22 (IBM, https:/ / www.ibm.com).

\section{Results}

We evaluated clinical features of the patients with and without history of neurosurgery (Table 1). Among the 27 patients with a history of neurosurgery, 5 (18.5\%) had no PSWCs on EEG, but only 64/1,121 (5.7\%) patients without history of neurosurgery demonstrated absence of PSWCs on EEG. However, we did not identify statistically significant differences for age at onset of CJD, sex, distribution of polymorphisms, disease duration, or positive rate of 14-3-3 protein or total tau protein in CSF.

Among the 27 sCJD patients with history of neurosurgery, 5 (18.5\%) had atypical CJD features, including no PSWCs on EEG during their illnesses (Table 2). Among the 5 patients with atypical sCJD, the median age $( \pm$ SD) at CJD onset was $65.4( \pm 9.6)$ years (range $49-75$ years); 4 were women and 1 was a man. Neurosurgery occurred during 1977-1993. The average time between neurosurgery and onset of CJD was $18.0( \pm 8.8)$ years (9-30 years), and the average time between onset of CJD and the appearance of the akinetic mutism or death was $17.0( \pm 7.7)$ months (6-28 months). Among the 5 patients with atypical sCJD, 2 patients were unclassified because we had no or insufficient information on dura mater graft during neurosurgery. The other 3 patients were determined to have SCJD without a history of dura mater-related neurosurgery. None of the 5 patients with atypical CJD had PRNP mutation, but all had 129MM.

DW-MRI was performed in 4/5 atypical patients and we noted hyperintensity lesions in various brain regions (Table 2). In patient 3 (Appendix Figure 1, panel A, https://wwwnc.cdc.gov/EID/article/26/6/181969-App1.pdf) and patient 4 (Appendix Figure 1, panel B), images showed hyperintensity lesions in bilateral thalamus and bilateral basal ganglia.

Autopsies were performed on $3 / 5$ patients with atypical CJD (patients 2, 3, and 5); all cases are

\begin{tabular}{|c|c|c|c|c|c|c|c|c|}
\hline $\begin{array}{l}\text { Patient } \\
\text { no. }\end{array}$ & $\begin{array}{l}\text { Age at CJD } \\
\text { onset, y/sex }\end{array}$ & $\begin{array}{c}\text { Year of } \\
\text { neurosurgery; } \\
\text { reason }\end{array}$ & $\begin{array}{c}\text { Time from } \\
\text { neurosurgery to } \\
\text { onset of CJD, y }\end{array}$ & Initial symptoms & $\begin{array}{c}\text { Disease } \\
\text { duration, } \\
\text { mo† }\end{array}$ & $\begin{array}{l}\text { Codon } 129 \\
\text { of } P R N P \\
\end{array}$ & $\begin{array}{l}\text { Lesions } \\
\text { on DW- } \\
\text { MRI }\end{array}$ & $\begin{array}{r}\text { Patholoc } \\
\text { findings }\end{array}$ \\
\hline 1 & $75 / \mathrm{M}$ & 1977; head trauma & 30 & Dementia & 11 & $\mathrm{MM}$ & $\mathrm{CC}, \mathrm{BG}$ & ND \\
\hline 2 & $49 / F$ & $\begin{array}{l}\text { 1985; subarachnoid } \\
\text { hemorrhage }\end{array}$ & 9 & Insomnia & 28 & MM & ND & MM2-T \\
\hline 3 & $75 / F$ & 1985; tumor & 14 & $\begin{array}{l}\text { Drowsiness, gait } \\
\text { disturbance }\end{array}$ & 6 & MM & BG, Th & $\begin{array}{l}\text { CJD- } \\
\text { MMiK }\end{array}$ \\
\hline 4 & $63 / F$ & 1985; tumor & 27 & Gait disturbance & 19 & MM & BG, Th & ND \\
\hline 5 & $64 / F$ & $\begin{array}{l}\text { 1993; subdural } \\
\text { hematoma }\end{array}$ & 10 & Visual impairment & 21 & MM & $\mathrm{CC}$ & MM2-C \\
\hline
\end{tabular}

${ }^{*}$ Among the patients with history of neurosurgery, average time (+ SD) from neurosurgery to onset of CJD was $18.0(+9.8)$ years and average age at neurosurgery was $47.2( \pm 10.2)$ years. However, among 22 patients with PSWCs on EEG, average time ( \pm SD) from neurosurgery to onset of CJD was $14.3( \pm 9.1)$ years and average age at neurosurgery was $58.0( \pm 12.2)$ years. We noted no statistically significant differences between patients with and without PSWCs on EEG in relation to time between neurosurgery and onset of CJD or in age at neurosurgery. BG, basal ganglia; CC, cerebral cortex; CJD, Creutzfeldt-Jakob disease; DW-MRI, diffusion weighted images on magnetic resonance imaging; MM, methionine homozygous; MM2-C, MM2cortical type sporadic CJD; MM2-T, MM2-thalamic type sporadic CJD; ND, not done; PRNP, prion protein gene; Th, thalamus. †Disease duration is the time between onset of CJD and the appearance of akinetic mutism or death. 
Table 3. Patients without history of neurosurgery who had no periodic sharp-wave complexes during duration of CJD and hyperintensity lesions in thalamus diffusion-weighted magnetic resonance imaging of the brain*

\begin{tabular}{|c|c|c|c|c|}
\hline Patient no. & Age at CJD onset, y/sex & Codon 129 of $P R N P$ & Hyperintensity lesions on DW-MRI & Pathology findings \\
\hline 1 & $58 / F$ & MM & CC, BG, Th & MM2+1 \\
\hline 2 & $65 / \mathrm{M}$ & MM & $\mathrm{CC}, \mathrm{Th}$ & $\mathrm{MM} 2+1$ \\
\hline 3 & $61 / F$ & MM & $\mathrm{CC}, \mathrm{BG}, \mathrm{Th}$ & ND \\
\hline 4 & $58 / \mathrm{M}$ & MV & BG, Th & MV2 \\
\hline 5 & $73 / F$ & MV & CC, Th & MV2 \\
\hline 6 & $65 / F$ & MV & CC, BG, Th & ND \\
\hline 7 & $65 / F$ & MV & $\mathrm{CC}, \mathrm{Th}$ & ND \\
\hline 8 & $75 / F$ & VV & BG, Th & VV2 \\
\hline 9 & 69/M & VV & BG, Th & VV2 \\
\hline 10 & $52 / \mathrm{M}$ & VV & $\mathrm{CC}, \mathrm{BG}, \mathrm{Th}$ & ND \\
\hline
\end{tabular}

reported in detail elsewhere (8,17-19). We assessed the subtypes of CJD with pathology and biochemical analysis of the brain and noted MM2-thalamic type in patient 2, CJD-MMiK in patient 3, and MM2-cortical type sCJD in patient 5 (Table 2). Patient 3 did not receive a dura mater graft according to neurosurgery records, and none was observed at autopsy.

Among patients who had not undergone neurosurgery, 64/1,121 showed no PSWCs on EEG, including 51 (79.7\%) with 129MM, 4 (6.3\%) with 129VV, and $9(14.1 \%)$ with $129 \mathrm{MV}$. Western blot of brain tissue performed on 25 patients revealed 5 with MM1 type CJD, 9 with MM2 type, 5 with MM2+1 type, 4 with MV2 type, and 2 with VV2 type. DW-MRI was available for 55/64 patients without PSWCs on EEG, 44 patients with $129 \mathrm{MM}, 8$ with $129 \mathrm{MV}$, and 3 with $129 \mathrm{VV}$. On DW-MRI, 10/55 patients had hyperintensity lesions in the thalamus (Table 3), 3 of whom had $129 \mathrm{MM}$, including 2 patients with MM2+1 type. Slight thalamic signal increase was observed in mediodorsal nuclei in all 3 patients with 129MM (Appendix Figure 2).

\section{Discussion}

We identified 27/1,155 (2.3\%) patients with diagnosed SCJD or unclassified CJD who had neurosurgery $\geq 1$ year before the onset of CJD. We also noted that more patients with history of neurosurgery had an absence of PSWCs on EEG than patients who had not had neurosurgery. Furthermore, 5/27 patients with history of neurosurgery had atypical clinical features, including no PSWCs on EEG for the duration of CJD. Of note, among 5 patients with 129MM, 2 had hyperintensity lesions in the thalamus on DWMRI, 1 of whom was confirmed to have CJD-MMiK by autopsy.

In our previous study, patients with acquired CJD-MMiK showed no PSWCs $\leq 1$ year after onset of CJD $(4,5)$. Among 3 autopsies for cases without PSWCs on EEG and a history of neurosurgery, 2 patients were determined to have definite MM2 type sCJD with atypical clinical features (Table 2), as previously reported (19-22). One had MM2-cortical form and the other had MM2-thalamic form. Our study proved 1 patient had CJD-MMiK (Table 2), which we reported previously $(8,17)$. Among the patients with history of neurosurgery, no PSWCs on EEG might suggest possibility of acquired CJD-MMiK.

The patient determined to have CJD-MMiK showed hyperintensity lesions in bilateral thalamus in addition to bilateral basal ganglia (Appendix Figure 1, panel A). Several patients with plaque type dCJD showed hyperintensity lesions in thalamus on DW-MRI or proton density-weighted MRI (5,23-25; K. Sakai et al., unpublished data). A previous study on MRI of sCJD showed that the absence of thalamic signal increase differentiated MM1 from other types of sCJD, but thalamic involvement was occasionally observed in MM2-cortical type sCJD (26). In our study, thalamic signal increase was observed in 3/44 patients with diagnosed SCJD, and $129 \mathrm{MM}$ patients without history of neurosurgery showed only slight signal increases in mediodorsal nuclei. These results suggest that hyperintensity lesions in the thalamus on DW-MRI in patients with 129MM might be a clinical marker of CJD-MMiK, but we cannot exclude the possibility of MM2-cortical type sCJD. Further MRI studies of plaque type dCJD cases with CJD-MMiK is ongoing.

According to a previous study on MRI of sCJD, the most characteristic MRI lesion patterns in the patients with MV2 and VV2 type sCJD, who were infected with V2 sCJD strain, shows predominant involvement of thalamus and basal ganglia (26). Because transmission of V2 sCJD strain to persons with $129 \mathrm{MM}$ causes CJD-MMiK (7-10), thalamic involvement frequently was observed, as we noted in the case we identified in this study.

One of the 2 atypical patients with history of neurosurgery, patient 4 (Table 2), did not have an 
autopsy but had signs and symptoms of CJD-MMiK. Patient 4 had bilateral hyperintensity lesions in thalamus on DW-MRI (Appendix Figure 1, panel B), no PSWCs on EEG, methionine homozygosity at codon 129 of PRNP, gait disturbance as an initial symptom, and long disease duration (19 months), similar to the features of the patients with plaque-type dCJD, suggesting that this patient had CJD-MMiK. However, no information about dura mater grafting at the neurosurgery is available for this patient.

We identified 1 patient with definite CJDMMiK and another patient with suspected CJDMMiK among 1,155 patients with diagnosed sCJD or unclassified CJD, suggesting that these 2 patients could have iatrogenic CJD. Several epidemiologic studies have shown no association between surgery and the onset of sCJD, but others show notable association when special conditions are met, such as lag periods $>20$ years between surgery and CJD onset and when patients are $<30$ years of age at the time of surgery $(27,28)$. For the 2 patients with confirmed and suspected CJD-MMiK in our study, patient 3 underwent neurosurgery at 61 years of age, 14 years before CJD onset. Patient 4 underwent neurosurgery at 36 years of age, 27 years before CJD onset. Both of these patients' illness onset fall into the range noted between surgical procedure to illness onset (1-30 years) among 154 patients with dCJD in Japan (29). Compared with other surgical procedures, neurosurgery followed by protracted survival is remarkably infrequent and therefore is difficult to study. Our results showed that few patients mimic the findings of MMiK type CJD, which might provide clues supporting the presence of a subgroup of CJD transmitted by neurosurgery after a long time lag without evidence of reuse of contaminated instruments on a CJD patient. An experimental research study provides strong support for an eventual transmission of CJD by general surgery in addition to neurosurgery (30), and high-quality epidemiologic research on neurosurgical risk for CJD is needed. Furthermore, we cannot find iatrogenic CJD patients who transmit M1 sCJD strain to persons with $129 \mathrm{MM}$ because the transmission would result in MM1 type neuropathology. A new method to detect transmission of M1 sCJD strain to persons with $129 \mathrm{MM}$ is needed.

Thus far, a patient with cadaveric pituitaryderived human growth hormone (hGH)-associated CJD in the United Kingdom was reported to have $129 \mathrm{MM}$, type i $\mathrm{PrP}^{\mathrm{Sc}}$, and kuru plaques (31), suggesting that patients without history of neurosurgery could develop CJD-MMiK. In that case, the patient received hGH through intramuscular injection. Another 2 patients with hGH-associated CJD were reported to have $129 \mathrm{MM}$ and type i $\operatorname{PrP}^{\mathrm{Sc}}(32,33)$. Unfortunately, detailed clinical information, including brain MRI, is not available for these patients, and we cannot compare the clinical manifestations with those for plaque type dCJD in Japan. Further studies for CJD-MMiK in patients with hGH-associated CJD are needed.

In conclusion, among 1,155 patients with diagnosed sCJD or unclassified type CJD, 2 patients with a history of neurosurgery had atypical clinical and neuropathologic features similar to acquired CJD-MMiK. A subgroup of sCJD mimicking features described in dCJD might have been transmitted by neurosurgery after long time lag. Hyperintensity lesions in the thalamus on DW-MRI in patients with 129MM might be a clinical marker of acquired CJD-MMiK. For epidemiologic surveillance studies, we propose clinical diagnostic criteria of sCJD potentially transmitted by neurosurgical procedures, which includes the criteria of possible, probable, or definite SCJD; methionine homozygosity at codon 129 of PRNP; no PSWCs on EEG; and hyperintensity lesions in the thalamus on DW-MRI.

\section{Acknowledgments}

The authors thank members of the Creutzfeldt-Jakob Disease Surveillance Committee, Creutzfeldt-Jakob disease specialists, participating physicians in prefectures in Japan, and the patients with Creutzfeldt-Jakob disease and their families for providing important clinical information.

This work was supported by a grant-in-aid from the Research Committee of Prion Disease and Slow Virus Infection, the Ministry of Health, Labour and Welfare of Japan to T.H., R.A., H.M., and M.Y.; a grant-in-aid from the Research Committee of Surveillance and Infection Control of Prion Disease, the Ministry of Health, Labour, and Welfare of Japan to T.K., Y.N., N.S., T.T., H.M., and M.Y.; and a grant-in-aid from the Research Committee of Molecular Pathogenesis and Therapies for Prion Disease and Slow Virus Infection, Agency for Medical Research and Development to T.H., A.K., H.M., and M.Y.

\section{About the Author}

Dr. Hamaguchi is an associate professor in the Department of Neurology and Neurobiology of Aging, Kanazawa University Graduate School of Medical Sciences, Kanazawa, Japan. His research interests include transmission of pathogenic protein, such as prion protein and amyloid $\beta$ protein, among humans. 


\section{References}

1. Prusiner SB. Prions. Proc Natl Acad Sci USA. 1998;95: 13363-83. https:/ / doi.org/10.1073/pnas.95.23.13363

2. Brown P, Brandel JP, Sato T, Nakamura $Y$, MacKenzie J, Will RG, et al. Iatrogenic Creutzfeldt-Jakob disease, final assessment. Emerg Infect Dis. 2012;18:901-7. https://doi.org/10.3201/eid1806.120116

3. Brown $\mathrm{P}$, Brandel JP, Preece M, Sato T. Iatrogenic Creutzfeldt-Jakob disease: the waning of an era. Neurology. 2006;67:389-93. https://doi.org/10.1212/01. wnl.0000231528.65069.3f

4. Noguchi-Shinohara M, Hamaguchi T, Kitamoto T, Sato T, Nakamura Y, Mizusawa H, et al. Clinical features and diagnosis of dura mater graft associated Creutzfeldt Jakob disease. Neurology. 2007;69:360-7. https:/ /doi.org/ 10.1212/01.wnl.0000266624.63387.4a

5. Yamada M, Noguchi-Shinohara M, Hamaguchi T, Nozaki I, Kitamoto T, Sato T, et al. Dura mater graftassociated Creutzfeldt-Jakob disease in Japan: clinicopathological and molecular characterization of the two distinct subtypes. Neuropathology. 2009;29:609-18. https://doi.org/10.1111/j.1440-1789.2008.00987.x

6. Kobayashi A, Asano M, Mohri S, Kitamoto T. Crosssequence transmission of sporadic Creutzfeldt-Jakob disease creates a new prion strain. J Biol Chem. 2007;282:30022-8. https://doi.org/10.1074/jbc.M704597200

7. Kobayashi A, Sakuma N, Matsuura Y, Mohri S, Aguzzi A, Kitamoto T. Experimental verification of a traceback phenomenon in prion infection. J Virol. 2010;84:3230-8. https://doi.org/10.1128/JVI.02387-09

8. Kobayashi A, Parchi P, Yamada M, Brown P, Saverioni D, Matsuura Y, et al. Transmission properties of atypical Creutzfeldt-Jakob disease: a clue to disease etiology? J Virol. 2015;89:3939-46. https:/ / doi.org/10.1128/JVI.03183-14

9. Kobayashi A, Parchi P, Yamada M, Mohri S, Kitamoto T. Neuropathological and biochemical criteria to identify acquired Creutzfeldt-Jakob disease among presumed sporadic cases. Neuropathology. 2016;36:305-10. https://doi.org/10.1111/neup.12270

10. Kobayashi A, Asano M, Mohri S, Kitamoto T. A traceback phenomenon can reveal the origin of prion infection. Neuropathology. 2009;29:619-24. https://doi.org/10.1111/ j.1440-1789.2008.00973.x

11. Nozaki I, Hamaguchi T, Sanjo N, Noguchi-Shinohara M, Sakai K, Nakamura Y, et al. Prospective 10-year surveillance of human prion diseases in Japan. Brain. 2010;133:3043-57. https://doi.org/10.1093/brain/awq216

12. Hamaguchi T, Sakai K, Noguchi-Shinohara M, Nozaki I, Takumi I, Sanjo N, et al. Insight into the frequent occurrence of dura mater graft-associated Creutzfeldt-Jakob disease in Japan. J Neurol Neurosurg Psychiatry. 2013;84:1171-5. https://doi.org/10.1136/jnnp-2012-304850

13. Satoh K, Tobiume M, Matsui $Y$, Mutsukura K, Nishida N, Shiga $Y$, et al. Establishment of a standard 14-3-3 protein assay of cerebrospinal fluid as a diagnostic tool for Creutzfeldt-Jakob disease. Lab Invest. 2010;90:1637-44. https://doi.org/10.1038/labinvest.2009.68

14. Schmitz M, Ebert E, Stoeck K, Karch A, Collins S, Calero M, et al. Validation of 14-3-3 protein as a marker in sporadic Creutzfeldt-Jakob disease diagnostic. Mol Neurobiol. 2016;53:2189-99. https:/ / doi.org/10.1007/s12035-015-9167-5

15. Kitamoto T, Ohta M, Doh-ura K, Hitoshi S, Terao Y, Tateishi J. Novel missense variants of prion protein in Creutzfeldt-Jakob disease or Gerstmann-Sträussler syndrome. Biochem Biophys Res Commun. 1993;191:709-14. https://doi.org/10.1006/bbrc.1993.1275
16. World Health Organization. Global surveillance, diagnosis, and therapy of human transmissible spongiform encephalopathies: Report of a WHO consultation, February 9-11, 1998. Geneva: the Organization; 1998.

17. Ishida C, Kakishima A, Okino S, Furukawa Y, Kano M, Oda Y, et al. Sporadic Creutzfeldt-Jakob disease with MM1-type prion protein and plaques. Neurology. 2003; 60:514-7. https:// doi.org/10.1212/01.WNL. 0000044403.41041.A4

18. Nozaki I, Hamaguchi T, Noguchi-Shinohara M, Ono K, Shirasaki H, Komai K, et al. The MM2-cortical form of sporadic Creutzfeldt-Jakob disease presenting with visual disturbance. Neurology. 2006;67:531-3. https:/ / doi.org/10.1212/01.wnl.0000228224.35678.60

19. Hamaguchi $\mathrm{T}$, Kitamoto $\mathrm{T}$, Sato $\mathrm{T}$, Mizusawa $\mathrm{H}$, Nakamura Y, Noguchi M, et al. Clinical diagnosis of MM2type sporadic Creutzfeldt-Jakob disease. Neurology. 2005;64:643-8. https://doi.org/10.1212/01.WNL. 0000151847.57956.FA

20. Parchi P, Capellari S, Chin S, Schwarz HB, Schecter NP, Butts JD, et al. A subtype of sporadic prion disease mimicking fatal familial insomnia. Neurology. 1999;52: 1757-63. https:/ / doi.org/10.1212/WNL.52.9.1757

21. Parchi P, Giese A, Capellari S, Brown P, Schulz-Schaeffer W, Windl O, et al. Classification of sporadic CreutzfeldtJakob disease based on molecular and phenotypic analysis of 300 subjects. Ann Neurol. 1999;46:224-33. https:/ / doi.org/ 10.1002/1531-8249(199908)46:2<224: AID-ANA12>3.0.CO;2-W

22. Krasnianski A, Meissner B, Schulz-Schaeffer W, Kallenberg K, Bartl M, Heinemann U, et al. Clinical features and diagnosis of the MM2 cortical subtype of sporadic Creutzfeldt-Jakob disease. Arch Neurol. 2006;63:876-80. https://doi.org/10.1001/archneur.63.6.876

23. Kretzschmar HA, Sethi S, Földvári Z, Windl O, Querner V, Zerr I, et al. Latrogenic Creutzfeldt-Jakob disease with florid plaques. Brain Pathol. 2003;13:245-9. https://doi.org/ 10.1111/j.1750-3639.2003.tb00025.x

24. Meissner B, Kallenberg K, Sanchez-Juan P, Ramljak S, Krasnianski A, Heinemann U, et al. MRI and clinical syndrome in dura mater-related Creutzfeldt-Jakob disease. J Neurol. 2009;256:355-63. https://doi.org/10.1007/ s00415-009-0026-Z

25. Wakisaka Y, Santa N, Doh-ura K, Kitamoto T, Ibayashi S, Iida $\mathrm{M}$, et al. Increased asymmetric pulvinar magnetic resonance imaging signals in Creutzfeldt-Jakob disease with florid plaques following a cadaveric dura mater graft. Neuropathology. 2006;26:82-8. https://doi.org/10.1111/ j.1440-1789.2006.00638.x

26. Meissner B, Kallenberg K, Sanchez-Juan P, Collie D, Summers DM, Almonti S, et al. MRI lesion profiles in sporadic Creutzfeldt-Jakob disease. Neurology. 2009; 72:1994-2001. https://doi.org/10.1212/

WNL.0b013e3181a96e5d

27. de Pedro-Cuesta J, Mahillo-Fernandez I, Calero M, Rábano A, Cruz M, Siden Å, et al.; EUROSURGYCJD Research Group. Towards an age-dependent transmission model of acquired and sporadic Creutzfeldt-Jakob disease. PLoS One. 2014;9:e109412. https://doi.org/10.1371/ journal.pone.0109412

28. López FJG, Ruiz-Tovar M, Almazán-Isla J, Alcalde-Cabero E, Calero M, de Pedro-Cuesta J. Risk of transmission of sporadic Creutzfeldt-Jakob disease by surgical procedures: systematic reviews and quality of evidence. Euro Surveill. 2017;22. https://doi.org/10.2807/1560-7917.ES. 2017.22.43.16-00806 
29. Ae R, Hamaguchi T, Nakamura Y, Yamada M, Tsukamoto T, Mizusawa H, et al. Update: dura mater graft-associated Creutzfeldt-Jakob disease-Japan, 1975-2017. MMWR Morb Mortal Wkly Rep. 2018;67:274-8. https://doi.org/10.15585/ mmwr.mm6709a3

30. Brown P, Gibbs CJ Jr, Rodgers-Johnson P, Asher DM, Sulima MP, Bacote A, et al. Human spongiform encephalopathy: the National Institutes of Health series of 300 cases of experimentally transmitted disease. Ann Neurol. 1994;35:513-29. https:// doi.org/10.1002/ana.410350504

31. Ritchie DL, Barria MA, Peden AH, Yull HM, Kirkpatrick J, Adlard P, et al. UK Iatrogenic Creutzfeldt-Jakob disease: investigating human prion transmission across genotypic barriers using human tissue-based and molecular approaches. Acta Neuropathol. 2017;133:579-95. https:/ / doi.org/10.1007/s00401-016-1638-x

32. Cali I, Cohen ML, Haik S, Parchi P, Giaccone G, Collins SJ, et al. Iatrogenic Creutzfeldt-Jakob disease with amyloid- $\beta$ pathology: an international study. Acta Neuropathol Commun. 2018;6:5. https://doi.org/10.1186/ s40478-017-0503-z

33. Duyckaerts C, Sazdovitch V, Ando K, Seilhean D, Privat N, Yilmaz Z, et al. Neuropathology of iatrogenic Creutzfeldt-Jakob disease and immunoassay of French cadaver-sourced growth hormone batches suggest possible transmission of tauopathy and long incubation periods for the transmission of Abeta pathology. Acta Neuropathol. 2018;135:201-12. https://doi.org/10.1007/ s00401-017-1791-x

Address for correspondence: Masahito Yamada, Department of Neurology and Neurobiology of Aging, Kanazawa University Graduate School of Medical Science, 13-1 Takara-machi, Kanazawa 920-8640, Japan; email: m-yamada@med.kanazawa-u.ac.jp

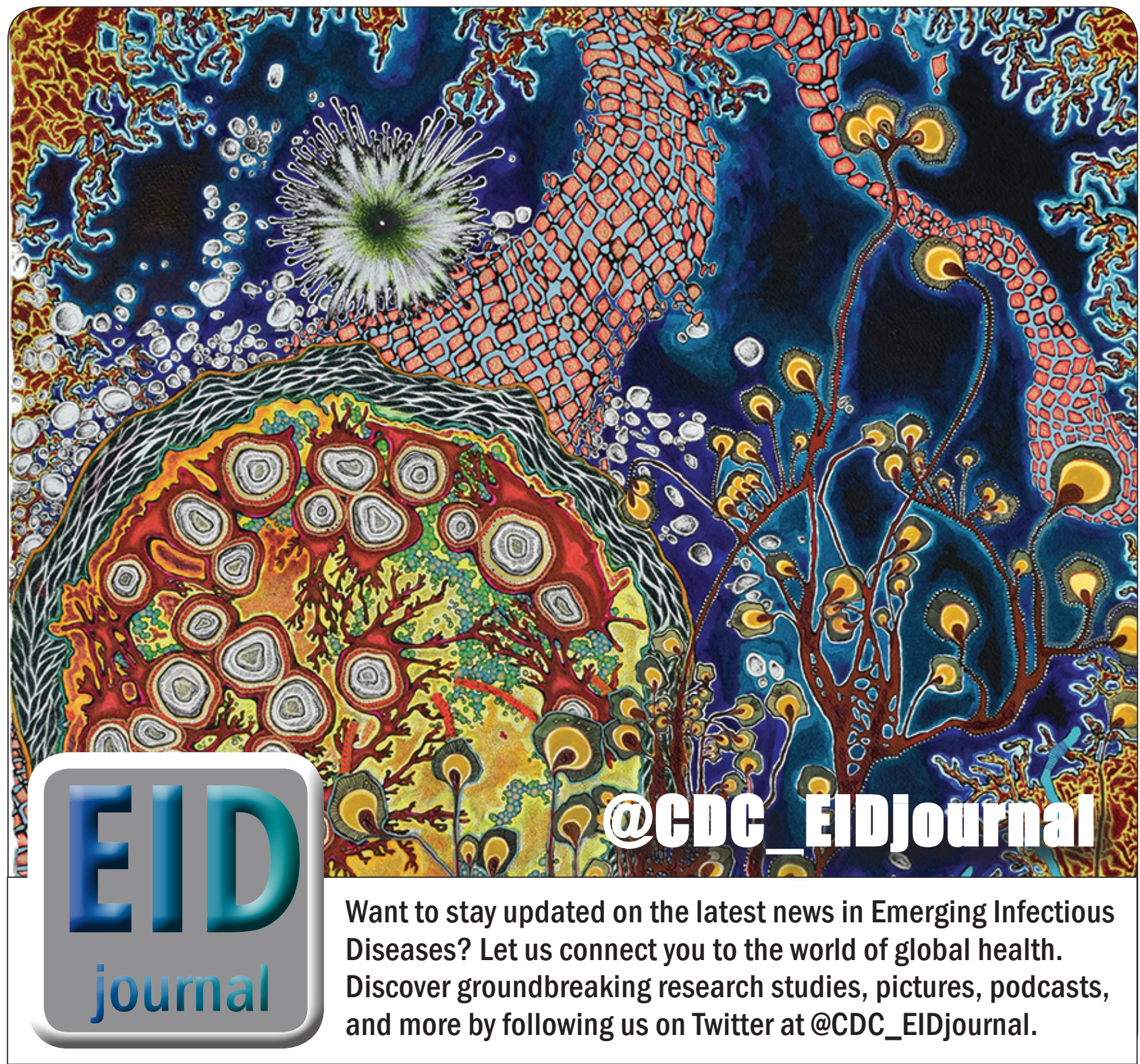

